\title{
Evaluation of Semantic Clusters
}

\author{
Rajeev Agarwal \\ Mississippi State University \\ Mississippi State, MS 39762 \\ USA \\ rajeev@cs.msstate.edu
}

\begin{abstract}
Semantic clusters of a domain form an important feature that can be useful for performing syntactic and semantic disambiguation. Several attempts have been made to extract the semantic clusters of a domain by probabilistic or taxonomic techniques. However, not much progress has been made in evaluating the obtained semantic clusters. This paper focuses on an evaluation mechanism that can be used to evaluate semantic clusters produced by a system against those provided by human experts.
\end{abstract}

\section{Introduction ${ }^{1}$}

Most natural language processing (NLP) systems are designed to work on certain specific domains and porting them to other domains is often a very timeconsuming and human-intensive process. As the need for applying NLP systems to more and varied domains grows, it becomes increasingly important that some techniques be used to make these systems more portable. Several researchers (Lang and Hirschman, 1988; Rau et al., 1989; Pustejovsky, 1992; Grishman and Sterling, 1993; Basili et al., 1994), either directly or indirectly, have addressed issues that assist in making it easier to move an NLP system from one domain to another. One of the reasons for the lack of portability is the need for domain-specific semantic features that such systems often use for lexical, syntactic, and semantic disambiguation. One such feature is the knowledge of the semantic clusters in a domain.

Since semantic classes are often domain-specific, their automatic acquisition is not trivial. Such classes can be derived either by distributional means or from existing taxonomies, knowledge bases, dictionaries, thesauruses, and so on. A prime example of the latter is WordNet which has been used to

${ }^{1}$ The author is currently at Texas Instruments and all inquiries should be addressed to rajeev@csc.ti.com. provide such semantic classes (Resnik, 1993; Basili et al., 1994) to assist in text understanding. Our efforts to obtain such semantic clusters with limited human intervention have been described elsewhere (Agarwal, 1995). This paper concentrates on the aspect of evaluating the obtained clusters against classes provided by human experts.

\section{The Need}

Although there has been a lot of work done in extracting semantic classes of a given domain, relatively little attention has been paid to the task of evaluating the generated classes. In the absence of an evaluation scheme, the only way to decide if the semantic classes produced by a system are "reasonable" or not is by having an expert analyze them by inspection. Such informal evaluations make it very difficult to compare one set of classes against another and are also not very reliable estimates of the quality of a set of classes. It is clear that a formal evaluation scheme would be of great help.

Hatzivassiloglou and McKeown (1993) cluster adjectives into partitions and present an interesting evaluation to compare the generated adjective classes against those provided by an expert. Their evaluation scheme bases the comparison between two classes on the presence or absence of pairs of words in them. Their approach involves filling in a YES-NO contingency table based on whether a pair of words (adjectives, in their case) is classified in the same class by the human expert and by the system. This method works very well for partitions. However, if it is used to evaluate sets of classes where the classes may be potentially overlapping, their technique yields a weaker measure since the same word pair could possibly be present in more than one class.

An ideal scheme used to evaluate semantic classes should be able to handle overlapping classes (as opposed to partitions) as well as hierarchies. The technique proposed by Hatzivassiloglou and McKeown does not do a good job of evaluating either of these. In this paper, we present an evaluation methodology which makes it possible to properly evaluate over- 
Table 1: Two Example Classes

\begin{tabular}{|l|l|}
\hline $\begin{array}{l}\text { Class A } \\
\text { (System) }\end{array}$ & $\begin{array}{l}\text { Class B } \\
\text { (Expert) }\end{array}$ \\
\hline \hline cat & horse \\
dog & cow \\
stomach & cat \\
pig & pig \\
cow & lamb \\
hair & dog \\
cattle & sheep \\
goat & mare \\
& cattle \\
& swine \\
& goat \\
\hline
\end{tabular}

lapping classes. Our scheme is also capable of incorporating hierarchies provided by an expert into the evaluation, but still lacks the ability to compare hierarchies against hierarchies.

In the discussion that follows, the word "clustering" is used to refer to the set of classes that may be either provided by an expert or generated by the system, and the word "class" is used to refer to a single class in the clustering.

\section{Evaluation Approach}

As mentioned above, we intend to be able to compare a clustering generated by a system against one provided by an expert. Since a word can occur in more than one class, it is important to find some kind of mapping between the classes generated by the system and the classes given by the expert. Such a mapping tells us which class in the system's clustering maps to which one in the expert's clustering, and an overall comparison of the clusterings is based on the comparison of the mutually mapping classes.

Before we delve deeper into the evaluation process, we must decide on some measure of "closeness" between a pair of classes. We have adopted the F-measure (Hatzivassiloglou and McKeown, 1993; Chincor, 1992). In our computation of the Fmeasure, we construct a contingency table based on the presence or absence of individual elements in the two classes being compared, as opposed to basing it on pairs of words. For example, suppose that Class A is generated by the system and Class B is provided by an expert (as shown in Table 1). The contingency table obtained for this pair of classes is shown in Table 2.

The three main steps in the evaluation process are the acquisition of "correct" classes from domain experts, mapping the experts' clustering to that generated by the system, and generating an overall measure that represents the system's performance when compared against the expert.
Table 2: Contingency Table for Classes A and B

\begin{tabular}{|l||c|c|}
\hline \multicolumn{1}{|c||}{} & \multicolumn{2}{c|}{ Expert } \\
\cline { 2 - 3 } & YES & NO \\
\hline \hline System - YES & 6 & 2 \\
\hline System - NO & 5 & 0 \\
\hline \hline
\end{tabular}

\subsection{Knowledge Acquisition from Experts}

The objective of this step is to get human experts to undertake the same task that the system performs, i.e., classifying a set of words into several potentially overlapping classes. The classes produced by a system are later compared to these "correct" classifications provided by the expert.

\subsection{Mapping Algorithm}

In order to determine pairwise mappings between the clustering generated by the system and one provided by an expert, a table of F-measures is constructed, with a row for each class generated by the system, and a column for every class provided by the expert. Note that since the expert actually provides a hierarchy, there is one column corresponding to every individual class and subclass provided by the expert. This allows the system's classes to map to a class at any level in the expert's hierarchy. This table gives an estimate of how well each class generated by the system maps to the ones provided by the expert.

The algorithm used to compute the actual mappings from the F-measure table is briefly described here. In each row of the table, mark the cell with the highest F-measure as a potential mapping. In general, conflicts arise when more than one class generated by the system maps to a given class provided by the expert. In other words, whenever a column in the table has more than one cell marked as a potential mapping, a conflict is said to exist. To resolve a conflict, one of the system classes must be re-mapped. The heuristic used here is that the class for which such a re-mapping results in minimal loss of F-measure is the one that must be re-mapped. Several such conflicts may exist, and re-mapping may lead to further conflicts. The mapping algorithm iteratively searches for conflicts and resolves them till no more conflicts exist. Note also that a system class may map to an expert class only if the F-measure between them exceeds a certain threshold value. This ensures that a certain degree of similarity must exist between two classes for them to map to each other. We have used a threshold value of 0.20 . This value is obtained purely by observations made on the F-measures between different pairs of classes with varying degrees of similarity. 
Table 3: Noun Clustering Results

\begin{tabular}{|c||c|c|c|}
\hline \multirow{2}{*}{ Expert } & \multicolumn{3}{c|}{ System } \\
\cline { 2 - 4 } & Precision & Recall & F-measure \\
\hline Expert A & 75.38 & 29.09 & 0.42 \\
\hline Expert B & 77.08 & 25.23 & 0.38 \\
\hline Expert C & 73.85 & 37.88 & 0.50 \\
\hline \hline
\end{tabular}

\subsection{Computation of the Overall F-measure}

Once the mappings have been determined between the clusterings of the system and the expert, the next step is to compute the F-measure between the two clusterings. Rather than populating separate contingency tables for every pair of classes, construct a single contingency table. For every pairwise mapping found for the classes in these two clusterings, populate the YES-YES, YES-NO, and NO-YES cells of the contingency table appropriately (see Table 2). Once all the mapped classes have been incorporated into this contingency table, add every element of all unmapped classes generated by the system to the YES-NO cell and every element of all unmapped classes provided by the expert to the NO-YES cell of this table. Once all classes in the two clusterings have been accounted for, calculate the precision, recall, and F-measure as explained in (Hatzivassiloglou and McKeown, 1993).

\section{Results and Discussion}

In one of our experiments, the 400 most frequent nouns in the Merck Veterinary Manual were clustered. Three experts were used to evaluate the generated noun clusters. Some examples of the classes that were generated by the system for the veterinary medicine domain are PROBLEM, TREATMENT, ORGAN, DIET, ANIMAL, MEASUREMENT, PROCESS, and so on. The results obtained by comparing these noun classes to the clusterings provided by three different experts are shown in Table 3. We have also experimented with the use of WordNet to improve the classes obtained by a distributional technique. Some initial experiments have shown that WordNet consistently improves the Fmeasures for these noun classes by about 0.05 on an average. Details of these experiments can be found in (Agarwal, 1995).

It is our belief that the evaluation scheme presented in this paper is useful for comparing different clusterings produced by the same system or those produced by different systems against one provided by an expert. The resulting precision, recall, and F-measure should not be treated as a kind of "gold standard" to represent the quality of these classes in some absolute sense. It has been our experience that, as semantic clustering is a highly subjective task, evaluating a given clustering against different experts may yield numbers that vary considerably. However, when different clusterings generated by a system are compared against the same expert (or the same set of experts), such relative comparisons are useful.

The evaluation scheme presented here still suffers from one major limitation - it is not capable of evaluating a hierarchy generated by a system against one provided by an expert. Such evaluations get complicated because of the restriction of one-to-one mapping. More work definitely needs to be done in this area.

\section{References}

Rajeev Agarwal. 1995. Semantic feature extraction from technical texts with limited human intervention. Ph.D. thesis, Mississippi State University, May.

Roberto Basili, Maria Pazienza, and Paola Velardi. 1994. The noisy channel and the braying donkey. In Proceedings of the ACL Balancing Act Workshop, pages 21-28, Las Cruces, New Mexico, July.

Nancy Chincor. 1992. MUC-4 evaluation metrics. In Proceedings of the Fourth Message Understanding Conference (MUC-4).

Ralph Grishman and John Sterling. 1993. Smoothing of automatically generated selectional constraints. In Proceedings of the ARPA Workshop on Human Language Technology. Morgan Kaufmann Publishers, Inc., March.

Vasileios Hatzivassiloglou and Kathleen R. McKeown. 1993. Towards the automatic identification of adjectival scales: Clustering adjectives according to meaning. In Proceedings of the 31st Annual Meeting of the Association for Computational Linguistics, pages 172-82.

Francois-Michel Lang and Lynette Hirschman. 1988. Improved portability and parsing through interactive acquisition of semantic information. In Proceedings of the Second Conference on Applied Natural Language Processing, pages 49-57, February.

James Pustejovsky. 1992. The acquisition of lexical semantic knowledge from large corpora. In Proceedings of the Speech and Natural Language Workshop, pages 243-48, Harriman, N.Y., February.

Lisa Rau, Paul Jacobs, and Uri Zernik. 1989. Information extraction and text summarization using linguistic knowledge acquisition. Information Processing and Management, 25(4):419-28.

Philip Resnik. 1993. Selection and Information: A Class-Based Approach to Lexical Relationships. Ph.D. thesis, University of Pennsylvania, December. (Institute for Research in Cognitive Science report IRCS-93-42). 